\title{
Aphid species specializing on milkweed harbor taxonomically similar bacterial communities that differ in richness and relative abundance of core symbionts
}

\section{Laramy Enders ( $\sim$ lenders@purdue.edu )}

Purdue University https://orcid.org/0000-0002-7569-1171

Thorsten Hansen

Purdue University

Kirsten Brichler

Southwest Minnesota State University

John Couture

Purdue University

Elizabeth French

Purdue University

\section{Research Article}

Keywords: Milkweed, aphid, symbiont, microbiome, host plant specialization, plant-insect interactions

Posted Date: January 19th, 2022

DOI: https://doi.org/10.21203/rs.3.rs-399030/v2

License: (c) (i) This work is licensed under a Creative Commons Attribution 4.0 International License.

Read Full License 


\section{Abstract}

Host plant range is arguably one of the most important factors shaping microbial communities associated with insect herbivores. However, it is unclear whether host plant specialization limits microbial community diversity or to what extent herbivores sharing a common host plant evolve similar microbiomes. To investigate whether variation in host plant range influences the assembly of core herbivore symbiont populations we compared bacterial diversity across three milkweed aphid species (Aphis nerii, Aphis asclepiadis, Myzocallis asclepiadis) feeding on a common host plant (Asclepias syriaca) using $16 \mathrm{~S}$ rRNA metabarcoding. Overall, there was significant overlap in taxa detected, but aphid species harbored bacterial communities that varied in terms of richness and relative abundance of key symbionts. However, bacterial community diversity did not vary with degree of aphid host plant specialization. Interestingly, the narrow specialist $A$. asclepiadis harbored significantly higher relative abundances of the facultative symbiont Arsenophonus compared to the other two aphid species. Although many low abundance microbes were shared across all milkweed aphids, key differences exist in symbiotic partnerships that could influence additional ecological variation, including variation in ant tending observed across milkweed aphid species via microbial induced changes to honeydew or defensive chemical profiles. Overall, this study suggests feeding on a common host plant can select for taxonomically similar microbiomes across herbivore species and highlights an intriguing potential role for variation in key aphid symbionts in host-plant interactions.

\section{Introduction}

Establishing partnerships with microbes is hypothesized to enhance the adaptive potential of the host organism [1-4]. In fact, both plants and insects harbor diverse microbial communities that provide unique advantages, including protection from stress and novel mechanisms for nutrient acquisition [47]. However, only recently have microbes come to be viewed as mediators of plant-insect interactions. From an insect perspective, microbes can be critical in facilitating or restricting the use of host plants by aiding in digestion of plant material and detoxification of anti-herbivore chemical defenses $[3,5,8]$. Soil and root-associated microbes also play essential roles in plant growth and defense against both above and belowground insect attackers $[6,7,9,10]$. Although microbes unquestionably impact plant-insect interactions, the ecological factors and selective forces shaping plant and insect-associated microbial communities remain poorly understood.

One factor thought to play a pivotal role in shaping insect herbivore microbiomes is host plant range or diet breadth, which can vary from a single species to hundreds of plant species [11-16]. Several plant associated factors, including nutritional quality and defensive chemistry, are known to influence insect microbial community dynamics by selecting for taxa that facilitate enhanced colonization and survival [17]. For example, associations with heritable bacterial symbionts have long been recognized as enabling phloem feeding insects to exploit an otherwise poor nutritional source $[17,18]$. Microbes are 
also hypothesized to contribute to variation in the capacity of insect herbivores to consume chemically defended plants [8]. Finally, feeding on different host plants can influence the composition of microbial communities associated with insect herbivores $[11,14,19,20]$. However, aside from a handful of wellcharacterized nutritional symbionts it is unclear to what extent insect microbial communities either directly or indirectly contribute to host plant adaptation and diet breadth. Furthermore, the mechanisms contributing to variation in herbivore microbiomes and ecological implications for plant-insect coevolution are not well understood [14].

Insect microbiomes consist of varying combinations of heritable symbionts and flexible pools of environmentally acquired microbes, but how host plant range shapes the taxonomic and functional diversity of these communities is [21-23]relatively unexplored [5, 24-26]. Variation in host plant range can influence the assembly of microbial communities and dynamics of symbiotic partnerships. For example, microbial communities of specialist herbivores may be less diverse relative generalist species that possibly require a larger microbial repertoire to successfully colonize highly variable hosts or simply acquire greater environmental microbial diversity from feeding on a broader host plant range [5, 24]. Alternatively, overlapping host plant range could result in selection for common microbes needed for successful colonization across different herbivore species due to similarities in nutritional ecology or defensive chemistry. Currently, it is unclear whether broader patterns associated with host plant use exist, such as a gradient in microbiome diversity from specialist to generalist herbivores or shifts in abundance of key heritable symbionts.

Heritable bacterial symbionts are hypothesized to expand the host plant range of sap-feeding insects, particularly aphids [26-28]. Signatures of host plant specificity have been detected in aphid microbiome composition [29-32]. Even within a single aphid species ecologically divergent biotypes specialized on different host plants can harbor distinct microbiomes that differ in taxonomic composition and frequency of symbionts $[25,33,34]$. In contrast, recent work suggests aphid phylogeny (i.e. species relatedness) and geographic isolation of populations are dominant factors predicting differences in bacterial communities, while host plant range may be less important overall [21-23]. However, aphid studies have primarily focused on the role microbial symbionts play in host plant use by highly polyphagous species, while much less is known about monophagous or oligophagous species. As a result, the relative importance of ecological factors such as host plant and geographic range in governing aphid symbiont community assemblages remains unclear.

The current study aims to address the following question: How does variation in host plant range shape the assembly of aphid symbiont communities? Aphids colonizing milkweed species in the subfamily Asclepiadaceae exhibit variation in host plant specialization [35] and thus represent an excellent system 
in which to investigate the role diet breadth plays in shaping aphid microbial community composition. Aphis nerii is considered a broad specialist feeding on over 50 plant species, including oleander, milkweed, and periwinkle. Aphis asclepiadis is a narrow specialist, feeding on less than 10 Asclepias spp., and Myzocalis asclepiadis is monophagous, feeding only on the common milkweed Asclepias syriaca [35, 36]. Interestingly, it is thought that milkweed aphid species have few microbial symbionts besides the universal obligate symbiont Buchnera, possibly due to the toxicity of milkweed defensive chemicals (e.g. cardenolides) which when ingested could make the internal aphid environment inhospitable for sustained microbial growth (e.g. via direct anti-microbial effects). However, few populations have been screened for symbionts and thus the bacterial community of milkweed aphids remains largely unexplored [37]. We therefore characterized the diversity and composition of bacterial communities using targeted amplicon sequencing of field collected aphids found naturally colonizing common milkweed ( $A$. syriaca). Specifically, we tested two alternative hypotheses regarding the role host plant range plays in shaping symbiont community assembly. First, symbiont diversity (i.e. bacterial species richness) is hypothesized to increase with degree of host plant specialization or increased diet breadth (A.nerii $>A$. asclepiadis $>M$. asclepiadis). Alternatively, overlap in host plant range is hypothesized to reduce differences in symbiont communities across aphid species (e.g. via purifying selection from exposure to similar plant defensive chemicals).

\section{Methods}

Aphid Field Collection \& Sample Preparation

Aphids were collected in July-Aug 2017 from a single host plant type, the common milkweed (A. syriaca), across 14 locations generally surrounding the Purdue campus (Supplementary Table S1). By sampling from a single host plant type across different locations we focus on detecting differences in heritable symbionts associated with variation in aphid diet breadth, and to a lesser extent environmentally acquired microbes. Locations were chosen based on the presence of large patches of common milkweed in open grassy fields away from roadsides. Distance between locations ranged from a minimum of $1 \mathrm{~km}$ to a maximum of $141 \mathrm{~km}$ (Supplementary Table S1). To avoid sampling heavily from single aphid clonal families (e.g. offspring from a single alate aphid colonizing a plant), aphids were collected from 3-5 separate plants (<20m apart) within a single location (e.g. from distinct milkweed patches when possible) and pooled together into a single sample. In some cases, 2 aphid species were found co-colonizing a single plant and thus single species versus mixed species samples were designated as such during data collection (Supplementary Table S1). Individual adult aphids were removed from milkweed leaves by hand using a paint brush, identified and separated by species, and then placed in Eppendorf storage tubes containing $95 \%$ ethanol. Only adult aphids were included because they have distinct morphological characteristics (e.g. body coloration) that allow for straightforward species identification, thus preventing mixed species samples and ensuring pools of aphids used for downstream analysis were separate species. All individuals collected from each separate plant at each location were stored at $-20 \mathrm{C}$ until 
further processing. The total number of aphids collected per species at a single location ranged from 10 individuals to $>50$ individuals.

For bacterial community profiling, groups of 5 individual aphids per species were selected from the total pooled sample per location for DNA extraction and sequencing. Total DNA was extracted from groups of 5 whole aphids using the Qiagen DNeasy kit following standard protocols. Aphids were not surface sterilized beyond storage in $95 \%$ ethanol and therefore, while bacterial DNA recovered is expected to be primarily from internal communities, not all external or cuticular bacterial DNA was eliminated and is expected to contribute to overall diversity estimates [38]. Total DNA concentration was measured using a Nanodrop spectrophotometer for all samples. In total 65 pools of aphids were processed and sequenced for microbiome analysis. Targeted amplicon sequencing was used to characterize the bacterial communities associated with the three milkweed aphid species ( $A$. nerii, $A$. asclepiadis, M. asclepiadis) across the 19 locations sampled. Library preparation and sequencing was performed at the University of Minnesota Genomics Core Facility on an Illumina MiSeq instrument (V3 cluster chemistry) following optimized methods [39] and using primers that target a 250bp segment of the V4 region of the 16s rDNA gene. Primers used were standard V4 region primers 515F-GTGCCAGCMGCCGCGGTAA and 806RGGACTACHVGGGTWTCTAAT. All sequence data is available on NCBI SRA database under project number PRJNA635683.

\section{Characterization \& Analysis of Aphid Bacterial Communities}

Sample demultiplexing was done by the University of Minnesota Genomics Center with Illumina software. Trimmomatic [v. 0.36; 32] and Cutadapt [ $v 1.13 ; 30]$ were used to remove adapters and primer sequences and low quality reads. All subsequent processing was performed in R ( $v 3.6 .3$ ) and Bioconductor ( $v 3.10)$. Trimmed reads were processed through the dada2 ( $v$ 1.14.1) pipeline by filtering and trimming based on read quality, inferring error rates, merging paired end reads, removing chimeras, and assigning taxonomy with the Silva reference database v. 132 [42]. Removal of very low abundance reads was done using a cut-off of fewer than 10 reads in $5 \%$ of the samples. Next, eukaryotic and mitochondrial sequences were removed. Lastly, individual samples with fewer than 2500 reads were removed and a final sample-level filtering step (i.e. Buchnera ASVs with $<1 \%$ reads within a sample were removed from individual samples) was applied to account for potential read contamination or "cross-talk" among samples that can occur in metabarcoding studies of microbial communities that are dominated by few high abundance symbionts (e.g. Buchnera - [43]). We applied the more conservative exclusion cut-off to Buchnera ASVs only in order to avoid loss of rare community members and minimize false positives resulting from mis-binning Buchnera sequences. A summary of raw sequencing results and processing steps can be found in Supplementary Table S2. 
Following sequence processing, all downstream analyses were run in R ( $v$ 3.6.3). All code for statistical analyses and generation of figures can be found in the Purdue University Github (https://github.itap.purdue.edu/LaramyEndersGroup/Milkweed-Aphid-Microbiome). Specifically, we compared standard alpha and beta diversity metrics using the phyloseq [v 1.30.0; 32] and vegan [v 2.5-6; 33] packages in $\mathrm{R}$ to determine the extent to which microbiomes varied in taxonomic composition and structure across aphid species and sampling locations. To compare species richness and evenness we used the Kruskal-Wallis test to test the effect of aphid species on diversity metrics, followed by the Wilcoxon Rank Sum test to identify significant differences among aphid species. Differences in the structure of bacterial communities across aphid species was assessed through PERMANOVA analysis of beta diversity (Unifrac, weighted Unifrac, Bray-Curtis) and visualized using Non-Metric Multidimensional Scaling (NMDS). Homogeneity of dispersion across aphid species' samples was also tested using PERMDISP. To identify differentially abundant bacterial sequences across aphid species we applied generalized linear mixed models to normalized read counts using DeSeq2 [v 1.26.0;34] and made pairwise comparisons between each aphid species. Normalized read counts account for variation in library size and were used to estimate relative abundance of each ASV. Finally, we compared strain level genetic differences in Buchnera and Arsenophonus ASVs using a phylogenetic approach by aligning sequences with the DECIPHER package [v 2.14.0; [47] and building Maximum-likelihood trees with the optim.pml(model= "GTR", rearrangement = "stochastic") function in the phangorn package in R [v 2.5.5; 35].

\section{Results}

Overall, most bacterial taxa identified through sequencing were found to occur in all three milkweed aphid species (Fig 1a). In total 45 amplified sequence variants (ASVs) were identified (Supplementary Table S3), 38 of which occurred in each species when all sampling locations were considered. Bacterial communities in general were dominated by the primary aphid symbiont Buchnera, which was in high relative abundance compared to the remaining taxa (Fig $1 \mathrm{~b}$ ). Among the non-Buchnera ASVs identified several well-known aphid facultative symbionts were found, including Arsenophonus, Serratia, and Hamiltonella, which occurred in all milkweed aphid species to varying degrees (Fig 1b). In a few samples Regiella, Ricketsiella, and Wolbachia were identified in very low abundance ( $<2$ reads in $10 \%$ of samples) and did not make the cutoff to be included in analysis (Supplementary Fig. S1). Much of the remaining low-abundance taxa identified are likely environmentally acquired from the plant host or surrounding soil (e.g. Pantoea, Pseudomonas, Streptococcus). The prevalence or proportion of samples that tested positive for each ASV also tended to vary across aphid species (Supplementary Table S3). For example, Buchnera was found in all samples, but Hamiltonella ranged from $13 \%(2 / 15)$ prevalence in $A$. asclepiadis samples to $100 \%$ (6/6) in M. asclepiadis and 30\% (13/43) in A. nerii samples. Serratia was also highly prevalent across all three species, ranging from $83 \%$ in $A$. nerii to $100 \%$ in $M$. asclepiadis samples. Interestingly, all three identified strains of Lactobacillus were found in $100 \%$ of $M$. asclepiadis samples but were only present in $50 \%$ or less of samples of the other two species. Cooccurring strains of Buchnera and Arsenophonus were also found to varying degrees in all three species, 
although it should be noted that we tested groups of aphids and it remains unknown whether individual aphids harbor different strains (i.e. multiple infections).

Although most ASVs were shared at a global level, several key differences indicate that each milkweed aphid species harbors distinct bacterial communities that vary in composition and abundance (Figs 1 \& 2, Supplementary Table S4). NMDS of Bray-Curtis dissimilarity between samples indicates that bacterial community structure is unique to each aphid species $(p<0.001$, Supplementary Table S5), but did not vary by location (Fig $2 \& 3$ ). Hierarchical clustering of samples by differences in community structure and relative abundance of each taxa further shows that each aphid species forms a unique group (Fig 3 ). In addition to differences in overall community structure there was significant variation in the bacterial species richness and evenness of communities found across the three aphid species (Fig 4, Supplementary Table S5). Interestingly, the broad specialist $A$. nerii had the lowest bacterial species richness and the more narrow specialist $A$. asclepiadis had the highest among the three species. Overall, results did not show a gradient in bacterial diversity (i.e. species richness) associated with diet breadth or degree of host-plant specialization across these three milkweed aphid species.

To further identify factors contributing to differences between aphid microbiomes we compared the relative abundance of individual taxa and variation in bacterial strain diversity (ASVs) (Supplementary Table S6; Figures $3 \& 5$ ). Differences in community structure were primarily driven by 1 ) variation in the composition of facultative symbionts and other low-abundance (non-Buchnera) bacteria (Figs 1b \& 3 ) and 2) the presence of Buchnera and Arsenophonus strains unique to each aphid species (Fig 5). Overall, A. nerii bacterial communities were dominated by Buchnera with few other symbionts in low abundance, which differs from the other two aphid species. Unique to $A$. asclepiadis was the overall higher Arsenophonus abundances, but lower Buchnera abundances compared to the other two species (Fig 1b). Each aphid species typically harbored a single dominant Buchnera strain in highest relative abundance, with some co-occurring strains at lower abundances (Figs 4 \& 5; Supplementary Table S3). Where communities differed the most was in Arsenophonus strain diversity and abundance (Figs $3 \& 5$ ). For example, there were 6 distinct Arsenophonus strains found within $A$. asclepiadis communities that ranged between $50-227$ fold higher relative abundance compared to $A$. nerii and $M$. asclepiadis. $A$. asclepiadis also harbored 5 distinct Buchnera strains across the locations sampled. In addition, Buchnera strains varied from $92.1 \%$ sequence similarity to several strains differing by only $1-2$ base pairs (>99.2\% similarity) (Supplementary Table S7). Arsenophonus strains were even more similar to each other, varying from $94.7-99.6 \%$ similarity (Supplementary Table S7).

\section{Discussion}

The prevailing view of host-associated microorganisms and their role in insect-plant coevolution is rapidly changing. Acquisition of beneficial microbes serves not only to expand the ecological niche of the 
host, but can add novel weaponry in the adaptive battle between plants and the herbivores that colonize them $[3,5,7,8]$. Furthermore, insects not only acquire microbes from host plants and the surrounding environment, but these microbial communities are intimately linked $[9,49,50]$. Disentangling the mechanisms driving variation in herbivore microbial communities and the ecological consequences for host plant specialization is therefore of great interest. In the current study, shifts were observed in the core microbiome of aphid species exhibiting varying degrees of host specialization within the milkweed family. Aphids feeding on milkweed share most bacterial taxa (Fig 1), but each species harbors unique populations that differ in strain diversity and relative abundance of core heritable symbionts, most notably Arsenophonus (Figs 1b, $3 \& 5$ ). However, broader patterns in bacterial symbiont diversity that scale with diet breadth were not observed (Figs $3 \& 4$ ). Instead, our results provide support for hypothesized effect of overlapping host plant range and common selective pressures (e.g. plant chemical defenses) leading to similarities in microbiomes across milkweed aphid species.

Host plant specificity can influence herbivore associated microbial communities on multiple levels, including causing changes in functional, taxonomic or strain level diversity or by altering the abundance of individual taxa. The microbiomes of generalists and specialist herbivores could also vary simply due to differences in the contribution of heritable versus environmentally acquired microbes, the latter being more variable and transient. However, diet may in fact generally be a poor predictor of insect bacterial community composition [51], with exceptions only in certain groups of herbivores [52]. For example, Lepidopteran larva have variable gut microbiomes that are likely shaped by the host plant they feed on, but general patterns associated with diet breadth are not observed due to the high turnover rate of these microbial communities [14]. In contrast, recent work in Costa Rican rolled-leaf beetles (Cephaloleia spp.) shows diet breadth is linked to microbiome diversity and community structure [24]. Previous research also indicates the composition of aphid microbial communities are structured by host plant $[11,15,16$, $20,25,27,32,34]$ and heritable bacterial symbionts are involved in expanding diet breadth $[26,28]$.

Our results show aphids specialized on a single plant family (Asclepiadaceae) that vary in diet breadth have similar bacterial communities on a taxonomic level, but differ in strain diversity and relative abundance of key symbionts (e.g. Arsenophonus). Horizontal transfer of facultative symbionts via host plants can occur in aphids [53,54], which could contribute to similarities in symbiont communities (i.e. shared ASVs) across different species that overlap in host range and/or naturally co-occur on plants. Another possible explanation is exposure to similar nutritional and chemical profiles could homogenize microbiomes of herbivore species feeding on the same host plants. The species in this study are specialists of the Asclepias (milkweed) family and therefore may have similar core symbionts due to exposure to closely related host plants. Additionally, phylogenetic relatedness can generally result in closely related aphid species harboring more similar microbiota than distantly related species [21]. However, one unexpected result was the occurrence of a single strain of Buchnera in all three aphid 
species (i.e. ASV 1; see Fig 2) even after stringent filtering for false positives. This is the dominant strain infecting A. nerii and is found in significantly higher abundance compared to the other two species (Fig 2, Supplementary Table S6). The current wealth of research on Buchnera shows this primary endosymbiont of aphids lives intracellularly, relies purely on vertical transmission, and has exhibited co-cladogenesis with aphid hosts over millions of years. It is unlikely this strain has been transferred across species, but it is also unclear why our dataset shows higher levels of sequencing "cross-talk" between samples than previously observed (e.g. [43]). One possible explanation could be that taxonomic classification using the 16s rRNA gene is unable to provide strain-level resolution for Buchnera in some cases (i.e. multiple strains with identical 16s sequences grouped as ASV1) and therefore additional genomic information is needed to distinguish unique strains found across milkweed aphid species.

Although milkweed aphid microbiomes were overall similar, key differences in symbiotic partnerships could contribute to additional ecological variation (e.g. ant tending, parasitism rates, predation). Interestingly, variation in symbiont relative abundance and strain diversity contributed most to differences observed across milkweed aphid microbiomes. In particular, Arsenophonus was found in higher abundance in A. asclepiadis compared to the other two species. Arsenophonus is a notorious shapeshifting insect symbiont, known best for reproductive manipulation of its host [55,56]. Most aphid facultative symbionts are found in much lower abundances compared to the obligate nutritional symbiont Buchnera, suggesting the unusually high abundance observed in $A$. asclepiadis could be linked to symbiont complementarity, as has occurred in other aphid species [57]. It is also possible Arsenophonus provides a general fitness boost, similar to what has been observed in the soybean aphid [58]. Finally, differences in symbiont populations could shape milkweed aphid-ant mutualisms, possibly via microbial induced changes to honeydew or emission of chemical compounds that mediate partner attraction. Previous work shows insect social partnerships not only uniquely influence each host's symbiotic microbiome [59], but that volatile organic compounds produced by aphid-associated microbes play a role in attracting ant mutualists [60]. Among milkweed aphids, $A$. asclepiadis is consistently tended by ants and benefits from enhanced protection from predators, while $A$. nerii is occasionally ant tended and $M$. asclepiadis appears to be a loner lacking ant friends [61]. Based on the current study an intriguing question arises; Does Arsenophonus mediate milkweed aphid-ant interactions and thus contribute to observed differences in ant attendance? Although Arsenophonus does not appear to influence the intensity of ant attendance in cowpea aphids [62], it is possible this symbiont has evolved a different ecological role in the case of $A$. asclepiadis. However, given the $16 \mathrm{~s}$ rRNA metabarcoding approach used in this study only provides relative abundances, Arsenophonus titer levels will need to be confirmed using additional methods such as quantitative PCR in order to take the first step towards addressing potential functions, including nutritional supplementation or aphid-ant interactions. Detection of additional facultative symbionts (e.g. Serratia) also warrants further investigation into symbiotic relationships and functional roles in milkweed aphid biology and ecology. 
The current study is limited in that aphids were collected from a single host plant (i.e. common milkweed) and generalist species with a host range outside the milkweed family (e.g. Myzus persicae) were not characterized due to low occurrence in the field. We sampled aphids from a single common host plant rather than multiple milkweed species in order to focus on identifying differences in core heritable symbionts (e.g. presence/absence of taxa, large shifts in relative abundance) and reduce variation introduced by environment and host plant differences. Consequently, our sampling design has limited ability to detect changes in environmentally acquired microbes and does not test for changes in microbiome composition induced by feeding on different host plant species. Imbalance in sampling across aphid species (e.g. fewer M. asclepiadis samples, Supplementary Table S1) resulting from natural variation in prevalence could also mean that some microbial variation was missed. Finally, while this study profiled only bacterial symbionts, additional microbes present in the broader aphid microbiome (e.g. fungi) may be affected by differences in host plant range. Additional studies are therefore needed to dive deeper into the role host plant species plays in shaping milkweed aphid symbiont community composition and function, especially potential links between plant defensive chemistry and microbiome assembly. In general, further research investigating the generalist-specialist gradient using herbivores that feed across multiple plant species and families is needed to clarify the extent to which diet breadth shapes microbial communities (e.g. [24]).

In summary, we did not find evidence for a gradient in bacterial community diversity associated with variation in diet breadth for milkweed specialized aphid species. Instead, our results suggest overlapping host plant range and shared hosts can result in selection for common microbes and thus highly similar microbiomes across species. However, milkweed aphids do harbor unique bacterial populations that vary in strain diversity and relative abundance of Arsenophonus, although a handful of other well-known aphid symbionts were also detected in low abundance. These findings suggest that while diet breadth may not be a major driver of divergence in overall taxonomic composition of aphid symbiont communities, factors such as strain level variation and differences in abundance offer alternative routes to generating adaptive potential. Further research is needed to determine the functional or ecological role played by milkweed aphid facultative symbionts and different co-occurring strains.

\section{References}

1. Barbosa P, Krischik VA, Jones CG (1991) Microbial mediation of plant-herbivore interactions. John Wiley \& Sons

2. Berenbaum MR (1988) Allelochemicals in insect-microbe-plant interactions; agents provocateurs in the coevolutionary arms race. Nov Asp Insect-Plant Interact 97-123

3. Mason CJ, Jones AG, Felton GW (2019) Co-option of microbial associates by insects and their impact on plant-folivore interactions. Plant Cell Environ 42:1078-1086 
4. Sugio A, Dubreuil G, Giron D, Simon J-C (2015) Plant-insect interactions under bacterial influence: ecological implications and underlying mechanisms. J Exp Bot 66:467-478

5. Hansen AK, Moran NA (2014) The impact of microbial symbionts on host plant utilization by herbivorous insects. Mol Ecol 23:1473-1496

6. Mendes R, Garbeva P, Raaijmakers JM (2013) The rhizosphere microbiome: significance of plant beneficial, plant pathogenic, and human pathogenic microorganisms. FEMS Microbiol Rev 37:634-663

7. Pineda A, Zheng S-J, van Loon JJ, et al (2010) Helping plants to deal with insects: the role of beneficial soil-borne microbes. Trends Plant Sci 15:507-514

8. Hammer TJ, Bowers MD (2015) Gut microbes may facilitate insect herbivory of chemically defended plants. Oecologia 179:1-14

9. Liu H, Macdonald CA, Cook J, et al (2019) An ecological loop: Host microbiomes across multitrophic interactions. Trends Ecol Evol 34:1118-1130

10. Grunseich JM, Thompson MN, Aguirre NM, Helms AM (2020) The Role of Plant-Associated Microbes in Mediating Host-Plant Selection by Insect Herbivores. Plants 9:6

11. Ferrari J, Darby AC, Daniell TJ, et al (2004) Linking the bacterial community in pea aphids with host-plant use and natural enemy resistance. Ecol Entomol 29:60-65

12. McLean AH, Parker BJ, Hrček J, et al (2016) Insect symbionts in food webs. Philos Trans R Soc B Biol Sci 371:20150325

13. Giron D, Dedeine F, Dubreuil G, et al (2017) Influence of microbial symbionts on plant-insect interactions. In: Advances in botanical research. Elsevier, pp 225-257

14. Jones AG, Mason CJ, Felton GW, Hoover K (2019) Host plant and population source drive diversity of microbial gut communities in two polyphagous insects. Sci Rep 9:1-11

15. Xu T-T, Jiang L-Y, Chen J, Qiao G-X (2020) Host Plants Influence the Symbiont Diversity of Eriosomatinae (Hemiptera: Aphididae). Insects 11:217. https://doi.org/10.3390/insects11040217

16. Qin M, Chen J, Xu S, et al Microbiota associated with Mollitrichosiphum aphids (Hemiptera: Aphididae: Greenideinae): diversity, host species specificity and phylosymbiosis. Environ Microbiol n/a: https://doi.org/10.1111/1462-2920.15391

17. Douglas AE (2013) Microbial brokers of insect-plant interactions revisited. J Chem Ecol 39:952961

18. Engel P, Moran NA (2013) The gut microbiota of insects-diversity in structure and function. FEMS Microbiol Rev 37:699-735 
19. Chung SH, Scully ED, Peiffer M, et al (2017) Host plant species determines symbiotic bacterial community mediating suppression of plant defenses. Sci Rep 7:1-13

20. Holt JR, Styer A, White JA, et al (2020) Differences in Microbiota between two Multilocus Lineages of the sugarcane Aphid (Melanaphis sacchari) in the continental United States. Ann Entomol Soc Am

21. McLean AH, Godfray HCJ, Ellers J, Henry LM (2019) Host relatedness influences the composition of aphid microbiomes. Environ Microbiol Rep 11:808-816

22. Jones RT, Sanchez LG, Fierer N (2013) A cross-taxon analysis of insect-associated bacterial diversity. PLoS One 8:e61218

23. Najar-Rodríguez AJ, McGraw EA, Mensah RK, et al (2009) The microbial flora of Aphis gossypii: Patterns across host plants and geographical space. J Invertebr Pathol 100:123-126. https://doi.org/10.1016/j.jip.2008.10.005

24. Blankenchip CL, Michels DE, Braker HE, Goffredi SK (2018) Diet breadth and exploitation of exotic plants shift the core microbiome of tropical herbivorous beetles. PeerJ Prepr 6:e26692v1

25. Gauthier J-P, Outreman Y, Mieuzet L, Simon J-C (2015) Bacterial communities associated with host-adapted populations of pea aphids revealed by deep sequencing of $16 \mathrm{~S}$ ribosomal DNA. PloS One 10:e0120664

26. Wagner SM, Martinez AJ, Ruan Y-M, et al (2015) Facultative endosymbionts mediate dietary breadth in a polyphagous herbivore. Funct Ecol 29:1402-1410

27. Guidolin AS, Cônsoli FL (2017) Symbiont diversity of Aphis (Toxoptera) citricidus (Hemiptera: Aphididae) as influenced by host plants. Microb Ecol 73:201-210

28. Leonardo TE, Muiru GT (2003) Facultative symbionts are associated with host plant specialization in pea aphid populations. Proc R Soc Lond B Biol Sci 270:S209-S212

29. Xu S, Jiang L, Qiao G, Chen J (2020) The Bacterial Flora Associated with the Polyphagous Aphid Aphis gossypii Glover (Hemiptera: Aphididae) Is Strongly Affected by Host Plants. Microb Ecol 79:971984. https://doi.org/10.1007/s00248-019-01435-2

30. Ferrari J, West JA, Via S, Godfray HCJ (2012) Population Genetic Structure and Secondary Symbionts in Host-Associated Populations of the Pea Aphid Complex. Evolution 66:375-390. https://doi.org/10.1111/j.1558-5646.2011.01436.x

31. Brady CM, Asplen MK, Desneux N, et al (2014) Worldwide Populations of the Aphid Aphis craccivora Are Infected with Diverse Facultative Bacterial Symbionts. Microb Ecol 67:195-204. https://doi.org/10.1007/s00248-013-0314-0 
32. Henry LM, Maiden MC, Ferrari J, Godfray HCJ (2015) Insect life history and the evolution of bacterial mutualism. Ecol Lett 18:516-525

33. Simon J-C, Carré S, Boutin M, et al (2003) Host-based divergence in populations of the pea aphid: insights from nuclear markers and the prevalence of facultative symbionts. Proc R Soc Lond B Biol Sci 270:1703-1712. https://doi.org/10.1098/rspb.2003.2430

34. Brady CM, White JA (2013) Cowpea aphid (Aphis craccivora) associated with different host plants has different facultative endosymbionts. Ecol Entomol 38:433-437.

https://doi.org/10.1111/een.12020

35. Blackman RL, Eastop VF (2008) Aphids on the world's herbaceous plants and shrubs, 2 volume set. John Wiley \& Sons

36. Züst T, Agrawal AA (2016) Population growth and sequestration of plant toxins along a gradient of specialization in four aphid species on the common milkweed Asclepias syriaca. Funct Ecol 30:547556

37. Zytynska SE, Weisser WW (2016) The natural occurrence of secondary bacterial symbionts in aphids. Ecol Entomol 41:13-26

38. Binetruy F, Dupraz M, Buysse M, Duron O (2019) Surface sterilization methods impact measures of internal microbial diversity in ticks. Parasit Vectors 12:268

39. Gohl DM, Vangay P, Garbe J, et al (2016) Systematic improvement of amplicon marker gene methods for increased accuracy in microbiome studies. Nat Biotechnol 34:942-949

40. Bolger AM, Lohse M, Usadel B (2014) Trimmomatic: a flexible trimmer for Illumina sequence data. Bioinformatics 30:2114-2120

41. Martin M (2011) Cutadapt removes adapter sequences from high-throughput sequencing reads. EMBnet J 17:10-12

42. Callahan BJ, McMurdie PJ, Rosen MJ, et al (2016) DADA2: high-resolution sample inference from Illumina amplicon data. Nat Methods 13:581-583

43. Jousselin E, Clamens A-L, Galan M, et al (2016) Assessment of a 16S rRNA amplicon Illumina sequencing procedure for studying the microbiome of a symbiont-rich aphid genus. Mol Ecol Resour 16:628-640. https://doi.org/10.1111/1755-0998.12478

44. McMurdie PJ, Holmes S (2013) phyloseq: an R package for reproducible interactive analysis and graphics of microbiome census data. PloS One 8:e61217

45. Dixon P (2003) VEGAN, a package of R functions for community ecology. J Veg Sci 14:927-930 
46. Love MI, Huber W, Anders S (2014) Moderated estimation of fold change and dispersion for RNAseq data with DESeq2. Genome Biol 15:550

47. Wright ES (2016) Using DECIPHER v2. 0 to analyze big biological sequence data in R. R J 8:

48. Schliep K, Potts AA, Morrison DA, Grimm GW (2016) Intertwining phylogenetic trees and networks. PeerJ Preprints

49. Hannula SE, Zhu F, Heinen R, Bezemer TM (2019) Foliar-feeding insects acquire microbiomes from the soil rather than the host plant. Nat Commun 10:1-9

50. Gomes SI, Kielak AM, Hannula SE, et al (2020) Microbiomes of a specialist caterpillar are consistent across different habitats but also resemble the local soil microbial communities. Anim Microbiome 2:1-12

51. Jones RT, Sanchez LG, Fierer N (2013) A cross-taxon analysis of insect-associated bacterial diversity. PLoS One 8:e61218

52. Colman DR, Toolson EC, Takacs-Vesbach CD (2012) Do diet and taxonomy influence insect gut bacterial communities? Mol Ecol 21:5124-5137

53. Pons I, Renoz F, Noël C, Hance T (2019) Circulation of the Cultivable Symbiont Serratia symbiotica in Aphids Is Mediated by Plants. Front Microbiol 10:764.

https://doi.org/10.3389/fmicb.2019.00764

54. Li Q, Fan J, Sun J, et al (2018) Plant-Mediated Horizontal Transmission of Hamiltonella defensa in the Wheat Aphid Sitobion miscanthi. J Agric Food Chem 66:13367-13377. https://doi.org/10.1021/acs.jafc.8b04828

55. Jousselin E, Cø eur d'Acier A, Vanlerberghe-Masutti F, Duron O (2013) Evolution and diversity of A rsenophonus endosymbionts in aphids. Mol Ecol 22:260-270

56. Nováková E, Hypša V, Moran NA (2009) Arsenophonus, an emerging clade of intracellular symbionts with a broad host distribution. BMC Microbiol 9:143

57. Chong RA, Moran NA (2018) Evolutionary loss and replacement of Buchnera, the obligate endosymbiont of aphids. ISME J 12:898-908

58. Wulff JA, White JA (2015) The endosymbiont Arsenophonus provides a general benefit to soybean aphid (Hemiptera: Aphididae) regardless of host plant resistance (Rag). Environ Entomol 44:574-581

59. Ivens AB, Gadau A, Kiers ET, Kronauer DJ (2018) Can social partnerships influence the microbiome? Insights from ant farmers and their trophobiont mutualists. Mol Ecol 27:1898-1914 
60. Fischer CY, Lognay GC, Detrain C, et al (2015) Bacteria may enhance species association in an ant-aphid mutualistic relationship. Chemoecology 25:223-232

61. Smith, R.A., Mooney, K.A., Agrawal, A.A. (2009) Coexistence of Three Specialist Aphids on Common Milkweed, Asclepias syriaca. Ecology 89:2187-2196

62. Katayama N, Tsuchida T, Hojo MK, Ohgushi T (2013) Aphid Genotype Determines Intensity of Ant Attendance: Do Endosymbionts and Honeydew Composition Matter? Ann Entomol Soc Am 106:761770

\section{Declarations}

Funding: This work was supported by start-up funds awarded to Dr. Laramy Enders from Purdue University.

Conflicts of interest/Competing interests: The authors have no conflicts of interest to declare that are relevant to the content of this article.

Ethics approval: Not applicable, research does not involve human subjects, their data or biological material

Consent to participate Not applicable, research does not involve human subjects, their data or biological material

Consent for publication Not applicable, research does not involve human subjects, their data or biological material

Availability of data and material: DNA sequencing data is available on the NCBI SRA database under project number PRJNA635683.

Code availability: All code for statistical analyses and figures are available at https://github.itap.purdue.edu/LaramyEndersGroup/Milkweed-Aphid-Microbiome. 
Authors' contributions: L.E. wrote and revised all drafts of the manuscript, conceived of and designed the study, and contributed to data collection. T.H., K.B. and J.C. contributed to data collection and sample processing, provided conceptual guidance and critical revisions/edits of manuscript drafts prior to final submission. E.F. performed all statistical analyses, contributed to data interpretation, developed figures, and provided critical revisions of manuscript drafts prior to final submission. All authors read and approved the final manuscript.

\section{Figures}

\section{Figure 1}

A) Venn Diagram showing the extent to which amplicon sequence variants (ASVs) are shared and which are unique to each milkweed aphid species from samples collected across all locations. $B) \log 10(x+1)$ normalized counts of bacterial taxa found across the three milkweed aphid species. If multiple strains were present they were grouped together under one genus. Very low abundant ASV (below $1 \%$ relative abundance) were grouped together into the "Other" category, except for known aphid symbionts (i.e. Hamiltonella) and significantly differentially abundant taxa (see Supplementary Tables S3 \& S4; Supplementary Fig. S2 for details). Differing letters indicate significantly differential abundance across species by DESeq2, with an adjusted $p=0.05$. NS $=$ not significant 


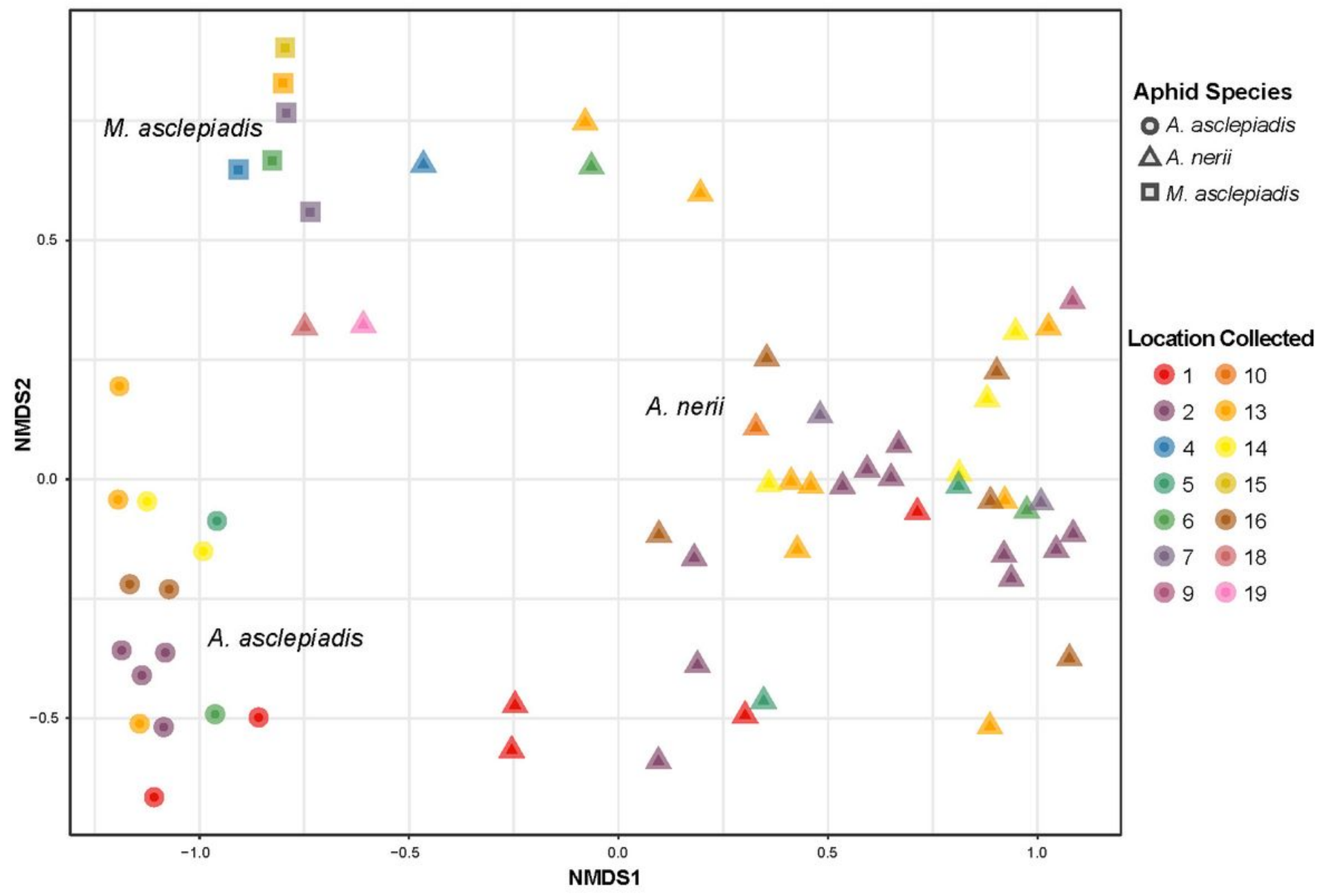

Figure 2

NMDS of Bray-Curtis dissimilarity showing how bacterial community structure varies across milkweed aphid species and location

Figure 3

Hierarchical clustering of all samples based on DESeq2 normalized read counts of each bacterial taxa 

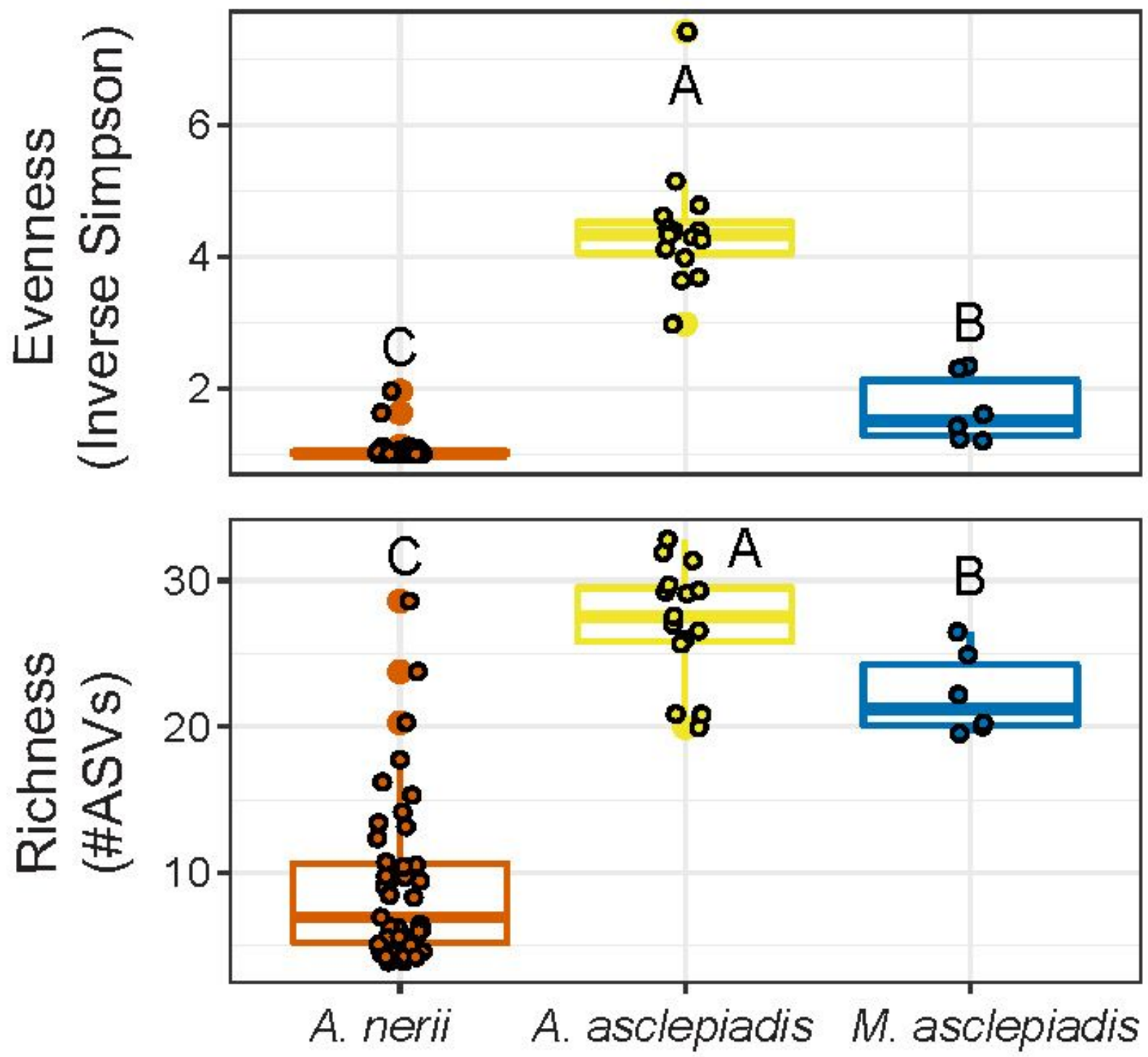

Figure 4

Species richness and evenness within aphid bacterial communities were compared across aphid species. Differing letters indicate significant differences at $p<0.05$ by Wilcoxon Rank Sum Test (Supplementary Table S5) 
A. Buchnera ASVs

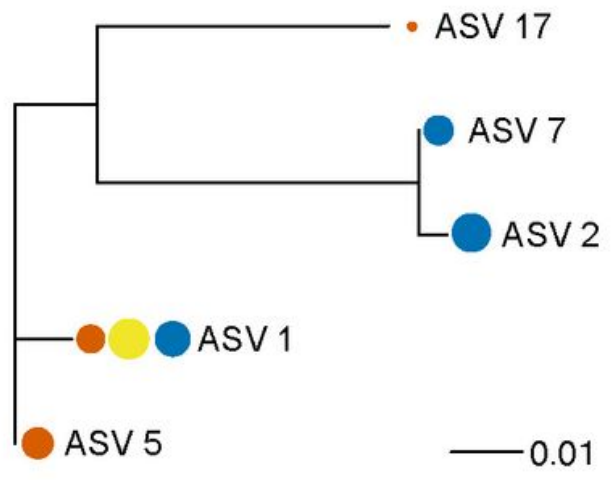

Average Normalized Counts

- 100
B. Arsenophonus ASVs

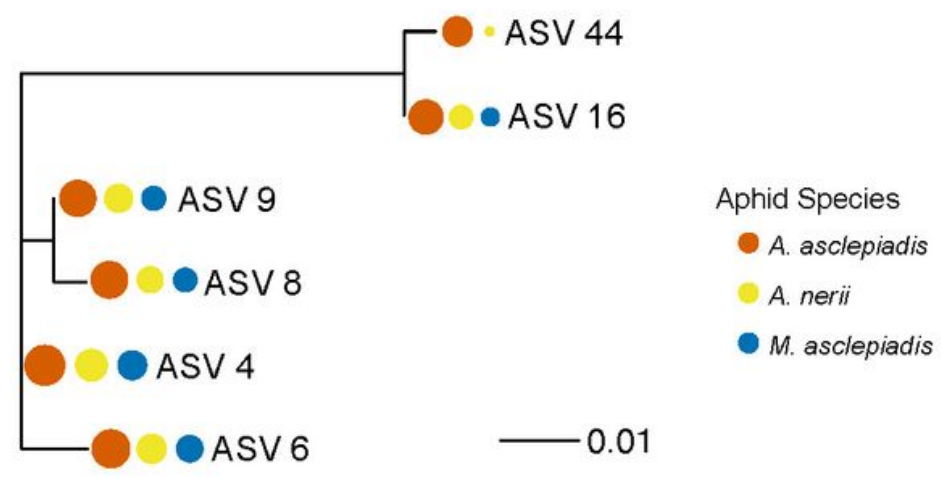

Average Normalized Counts

$1 \bigcirc 10 \bigcirc 100 \bigcirc 1000$

\section{Figure 5}

Genetic variation and differences in relative abundance of Buchnera and Arsenophonus strains (ASVs) identified in the three milkweed aphid species. All Buchnera and Arsenophonus ASVs except ASV 49 were differentially abundant (adjusted $p<0.05$ ) in at least one species comparison by DESeq2 (Supplementary Table S6)

\section{Supplementary Files}

This is a list of supplementary files associated with this preprint. Click to download.

- SupplementaryFigures.R1.docx

- SupplementaryTablesR2.xIsx 